\title{
Transplacentally transmitted organophosphorous compound poisoning in a newborn : Acase report and narrative review of literature.
}

\author{
Nargis Ara Begum ${ }^{1 *}$, Mohammad Omar Faruq ${ }^{2}$, Shahnaz Parvin Siddiqua ${ }^{3}$, Runa Laila ${ }^{3}$, Suman Sharker ${ }^{4}$, \\ Shirin Akter ${ }^{3}$

\begin{abstract}
:
Organophosphorous compound (OPC) poisoning in the newborn is a medical emergency but completely treatable condition. Early recognition and treatment is of paramount importance in preventing fatality. A newbornmale of severe perinatal asphyxia was admitted in our neonatal intensive care unit (NICU) with a history of maternal OPC poisoning 31 hours before delivery. We treated the case on urgent basis, had stormy neonatal course and finally baby went home safely. OPC poisoning in newborn is a rare occurrence in the tropics. We report a rare case of transplacentally transmission of OPC poisoning innewborn and it is going to be $3^{\text {rd }}$ and latest such reported case across the world upon
\end{abstract} \\ literature search.
}

Keywords: Organophosphorous compound, newborn, transplacental transmission.

\section{Introduction}

Organophosphates are widely used insecticides but still poses a serious health threat for all age groups. Common route of poisoning are ingestion, inhalation, through skin and mucous membrane and very rarely transplacental. Worldwide there was debate regarding this rare route of transplacental transmission. Neonatal organophosrouscompound (OPC) poisoning is a very rare event and only few cases have been reported so far. ${ }^{1-3}$ Some of the cases reported as babies born to mothers who had OPC poisoning by ingestion either suicidal or homicidal. Transplacental transmission of OPC poisoning is an example of such event. The rapidity of onset of symptoms of OPC poisoning depends on the dose, route of exposure and the compound potency. The clinical presentations of OPC poisoning in the newborn are respiratory difficulty, copious oral secretion, diarrhea, lethargy, jitteriness and seizure. The manifestations often simulate neonatal sepsis and leads to diagnostic dilemma. Careful clinical examination and early intervention is

1. Senior Consultant, Department of Pediatrics and Neonatology United Hospital Ltd.Dhaka1212, Bangladesh

2. Chief Consultant, GICU and Emergency, United Hospital Ltd.Dhaka1212, Bangladesh

3. Junior Consultant,Department of Pediatrics and Neonatology, United Hospital Ltd. Dhaka1212, Bangladesh.

4. Specialist, Department of Pediatrics and Neonatology United Hospital Ltd.Dhaka1212, Bangladesh

\section{*Corresponding Author:}

Dr. Nargis AraBegum

FCPS (Paediatrics), MD (Neonatology) Senior Consultant

Department of Pediatrics and Neonatology United Hospital Ltd.

Dhaka1212, Bangladesh

Cell phone: +8801914001148

E-mail: nargisdr@yahoo.com mandatory to treat the case. Confirmation can be done by low level of plasma cholinesterase, more commonly pseudocholinesterase level. Mother's serum or breast milk cholinesterase level could add in confirming the diagnosis. ${ }^{4,5}$ Treatment guideline includes general supportive measures like airway control, adequate oxygenation, ventilation, cardiovascular support. Mainstay of medical therapy include specific antidotes atropine, pralidoxime and benzodiazepins.

Here wereport acute toxicity of OPC in a newborn acquired transplacentally from pregnant mother who consumed OPC with suicidal intent. There were only 2 cases reported in literature worldwide ${ }^{1,6}$. To our knowledge, this is the $3^{\text {rd }}$ documented case and presented as severe perinatal asphyxia requiring artificial ventilation at birth, treated in time and was successfully discharged.

\section{Case Report:}

A 25 years old multigravida mother with uneventful pregnancy was hospitalized at 34 weeks of gestation with acute toxic manifestation of OPC poisoning due to suicidal intent at 31 hours before delivery. She was admitted directly in intensive care unit (ICU) and was treated by supportive therapy and specific antidote (atropine and pralidoxime). A preterm, male baby weighing 2250 gm (low birth weight) was delivered by caesarean section on the day of her admission. Baby was severely asphyxiated (apgar score $3 / 10$ at $1 \mathrm{~min}$ to $5 / 10$ at 5 minutes) and meconium stained at birth. The baby was apnoic, cyanosed and bradycardic. So, extensive resuscitation was needed along with endotracheal intubation (ET). Then the baby was shifted to neonatal intensive care unit (NICU) and was placed on mechanical ventilator. The baby required low setting in ventilation (rate 25, PIP 13, PEEP 5, fIO2- 30\% in SIMV-PC mode), SpO2 maintained 98-99\%. Clinical examination showed the baby was hypotonic, lethargic and bradycardic. Frequent ET suctioning was needed for copious bronchial secretion. Capillary refill time was delayed with mottled skin. The pupils were pin point, 
bilaterally constricted with no reaction to light. Anthropometric measurements were age appropriate. $1^{\text {st }} \& 2^{\text {nd }}$ heart sound were audible, air entry equal on both lungs and non-distended soft abdomen. Other systemic examination revealed normal.

In the NICU, in view of pin point pupil and bradycardia, excessive bronchial secretion, cholinergic overactivity was considered. Inj Atropine was started at $0.05 \mathrm{mg} / \mathrm{kg}$ intravenously over 15 minutes, maintained at $0.02 \mathrm{mg} / \mathrm{kg} / \mathrm{hr}$ and continued for 5 days. The baby showed initial clinical improvement with the disappearance of cholinergic signs, heart rate improved to more than 120 per minute as well pupillary size to increase. Also patient started having hypotension requiring inotrope support and had increased oxygen requirement. Echocardiography at that time revealed moderate persistent pulmonary hypertension of newborn (PPHN) with patent ductus arteriosus (PDA). The baby required fluid restriction and supportive therapy. The baby also developed muscle twitching \& fasciculation for which infusion of inj. Pralidoxime was started at $10 \mathrm{mg} / \mathrm{kg} / \mathrm{hour}$ at about 48 hours of life and continued for next 7 days. The baby had raised temperature of $100-101{ }^{\circ} \mathrm{F}$ but septic workup was normal.All biochemical parameters were normal. Acetylcholinesterase (AChE) level was very low (63\% lower than base line normal limit). With gradual improvement of general condition in the $2^{\text {nd }} \mathrm{wk}$ of life, there was sudden deterioration of respiratory status with jerky respiration and development of generalized convulsion. Anticonvulsant therapy was given and convulsion was controlled. CSF study was normal and ultrasonography of brain was unremarkable. As the respiratory effort improved, baby was gradually weaned from ventilator to continuous positive airway pressure (CPAP) and then to oxygen by face mask. By the time, repeat AChE level became normal. The baby was vitally stable, clinically well and alert, tolerated oral feed. The baby was discharged on day 15 of life, in room air with oralfeed.

\section{Discussion with narrative review.}

Organophosphate (OP) poisoning is potentially fatal. Prenatal OPC exposure can affect fetal development. Newborns exposed to maternal OPC during pregnancy has revealed growth disturbance, metabolic disorders, and CNS morbidity but no cardiac sequelae ${ }^{7}$. Suggested explanation is that the cardiac tissue can withstand the damage more efficiently in the fetal period and the changes may be transient.

OPCs are well known neurotoxin, which crosses the placental barrier and inhibits acetylcholine esterase, resulting buildup of acetylcholine in neuronal junction causing severeneurotoxicity. ${ }^{89}$ Fetus and newborn are more susceptible to neurotoxicity after exposure to OPC than adults $^{1-3}$. This neurotoxicity results in lower threshold for neonatal convulsion. ${ }^{10}$ The cause behind acute neurotoxicity in newborn and fetus is immaturity of the microsomal enzyme system and greater permeability of blood brain barrier. ${ }^{11,12}$ Developmental neurotoxicity studies have shown prenatal exposure may be associated with increased risk of developmental disorder, delay in cognition and attention deficit in childhood ${ }^{13-15}$.
Organophosphorus compounds inhibit acetylcholinesterase in the central and peripheral nervous system causing an increase in acetylcholine with the resultant muscarinic, nicotinic and central signs. ${ }^{4}$ Muscarinic signs are increased bronchial secretions, excessive sweating, salivation, lacrimation, meiosis, bronchospasm, vomiting, involuntary passage of stool and urine. Cardiac manifestations comprise bradycardia, hypotension and QT prolongation. Stimulation of the nicotinic receptor results in twitching, fasciculation, muscle weakness and flaccid paralysis. Stimulation of sympathetic ganglia leads to hypertension and tachycardia. Central signs include central nervous system (CNS) depression /convulsion and coma. ${ }^{16}$ Bradycardia, which was aprominent sign in our patient is found only in 15-20\% infant. Muscle fasciculation and seizures occur in one-fourth of all patients. In contrast toadults, CNS depression manifesting as coma, stupor, hypotonia and muscle weakness is seen in $90 \%$ children. ${ }^{17}$

Nicotinic and central muscarinic effects are predominant in neonates, unlike adults and children where only muscarinic effects are seen ${ }^{18}$. Our patient had history of severe perinatal asphyxia at birth requiring immediate ventilation, this effect was attributed by marked central muscarinic effect. In addition, transplacental passage of atropine following maternal atropinization might have contributed to perinatal asphyxia of newborn at birth. CNS manifestation of altered sensorium, recurrent seizure, cessation of spontaneous respiration and hypotension which the baby had, indicative of hypoxemic-ischemic encephalopathy (HIE), contributed by mixture of nicotinic, muscarinic and central effect.

OP compounds cause 3 types of paralysis: a)Type I- acute paralysis due to continuous depolarization of neuromuscular junction, b) Type II- Intermediate syndrome which develops 24-96 hours after initial improvement, presents as respiratory distress and weakness of proximal muscles, neck and trunk. Neuromuscular transmission defect or toxin induced muscle instability may be responsible for type II paralysis c) Type III-organophosphorous induced delayed polyneuropathy (OPIND), after (2-3) weeks of OPC exposure involves distal limbs but spares proximal muscles, trunk, neck. It's a rare event, may persists weeks to years ${ }^{19}$. Ourcase initially showed Type I paralysis but later progressed to Type II paralysis.

The diagnostic hallmark of OP poisoning is reduction in serum and RBC cholinesterase activity. RBC acetyl-cholinesterase activity is considered more sensitive and specific for OPC poisoningl. A 50\% or greater decrease below normal laboratory value is consistent with the diagnosis of OPC poisoning. ${ }^{20}$

Therapy is aimed at supporting ventilation as respiratory failure is the usual cause of death, aspiration pneumonia and hypotension are also encountered as possible causes.

Atropine is the mainstay of therapy, and can reverse the life threatening features of this acute poisoning. Atropine only blocks muscarinic effects. The recommended dose is 0.05 $\mathrm{mg} / \mathrm{kg}$ intravenous followed by 0.02 to $0.05 \mathrm{mg} / \mathrm{kg}$ every $5-10$ minutes till complete atropinization is achieved. A continuous infusion of atropine at 0.02 to $0.08 \mathrm{mg} / \mathrm{kg} /$ hour has also been 
used to maintain a steady atropinized state ${ }^{21}$ Cholinesterasere activators, oximes reverse both the nicotinic and muscarinic effects. Oximes can be highly effective in restoring skeletal muscle strength, reversing fasciculation and improving diaphragmatic weakness. It should be administered as an intravenous infusion over 30 minutes in a dose of $25-50 \mathrm{mg} / \mathrm{kg}$ within 24-48 h of exposure. The dose may be repeated after 1-2 hours then at 10-12 h intervals if cholinergic signs recur. ${ }^{22}$

Diazepam and other benzodiazepines are widely used for the treatment of OP induced seizures, restlessness and agitation. Recently, a quaternary ammonium compound glycopyrrolate has been used as an antidote to OP poisoning. It is as effective as atropine but has less tachycardia and fewer CNSeffects. ${ }^{23}$

Newer forms of therapies in OP poisoning are under active research. One small uncontrolled study from Iran concluded that the infusion of sodium bicarbonate significantly reduced total hospital stay, total atropine requirement and the need for intensive care therapy. ${ }^{24}$ Magnesium sulphate, fresh frozen plasma, antioxidants, Organophosphorus hydrolases and galyclidine(NMDA receptor antagonist) are all potential forms of therapies for thefuture..$^{25-29}$

\section{Conclusion:}

Transplacental transmission of organophosphorous poisoning is confirmed in our newborn baby. This is the $3^{\text {rd }}$ reported case across the world. Immediate supportive and treatment with specific antidotes could save the newborn, but patient needs close observation and follow-up to assess future neurodevelopment and long term outcome.

\section{REFERENCES:}

1. Jajoo M, Saxena S, Pandey M. Transplacentally acquired organophosphorus poisoning in a newborn: case report. Ann Trop Paediatr. 2010;30:137-139. DOI: https://doi.org/10.1179/ 14653 2810X 12703902516202

2. Samarawickrema N, PathmeswaranA, Wickremasinghe R, PeirisJohn R, KarunaratnaM, Buckley $\mathrm{N}$ et al. Fetal effects of environmental exposure of pregnant women to organophosphorus compounds in a rural farming community in Sri Lanka. ClinToxicol (Phila).2008;46:489-495. DOI: https://doi.org/10.1080/ 155636 50701837030

3. Abdulla EM, Hadidi MS, Alhadidi N, Al-NsourTS, HadidiKA. Agricultural and horticultural pesticides fatal poisoning; the Jordanian experience 1999-2002. J Clin Forensic Med. 2006; 13:304-307.DOI: https://doi.org/10.1016/j.jcfm.2006.06.011

4. Kaur I, JayashreeK, Hiranandani M, SinghiSC. Severe organophosphate poisoning in a neonate. Indian Pediatr.1996; 33: 517- 519 .

5. Choudhry VP, JallaliAJ, HaiderG, Aram GN, Ghani AR Organophosphorous poisoning. Indian $\mathrm{J}$ Pediatr. 1987; 54: 427-30.doi: 10.1007/BF02748933.

6. Sarkar S, NarangA, Singh S. Transplacentally acquired Carbamate Insecticide (Baygon) Poisoning in a Neonate. Indian Pediatrics. 1994; 31: 343-46.

7. Dordevic, VujicA, Jovanovic B, DordevicG, Sazdanovic M, SazdanovicP. Neonatal outcome following exposure $t$ o organophosphorus pesticides. Arch BiolSciBelgrade2010;62:1-8.
8. Gupta RC. Carbofuran toxicity. J Toxicol Environ Health. 1994;43:383-418.doi:10.1080/15287399409531931.

9. Ahlbom J, FredrikssonA, Eriksson P. Exposure to an organophosphate (DFP) during a defined period in neonatal life induces permanent changes in brain muscarinic receptors and behavior in adult mice. 1995. Brain Res 677: 13-19.

10. Wurpel JN, HirtPC, BidansetJH. Amygdala kindling in immature rats: proconvulsanteffect of the organophosphate insecticidechlorpyrifos. Neurotoxicology 1992; 14(4):429-36.

11. 2002 web report of the IEH. A review of the effects of low-level exposure to organophosphate pesticide on fetal \& childhood health. Institute for environmental health, 2002, web reportW11.

12. Ginsberg G, Hattis D, Sonawane B. Incorporating pharmacokinetic differences between children and adults in assessing children's risks to environmental toxicants. ToxicolApplPharmacol 2004; 198: 164-183.http://doi.org/10.1016/j.taap.2003.10.01

13. Rohlman DS, ArcuryTA, QuandtSA, Lasarev M, Rothlein J, et al. Neurobehavioral performance in preschool children from agricultural and non-agricultural communities i n Oregon and North Carolina. Neurotoxicology 2005; 26:589-598. https://doi.org/10.1016/j.neuro.2004.12.002

14. Grandjean P, Harari R, Barr DB, DebesF. Pesticide exposure and stunting as independent predictors of neurobehavioral deficits in Ecuadorian school children. Pediatrics 2006; 117:e546-556.

15. Eaton DL, Daroff RB, Autrup H, Bridges J, Buffler P, Costa LG et al. Review of the toxicology of chlorpyrifos with an emphasis on human exposure and neurodevelopment.Crit Rev Toxicol 2008;38 Suppl 2:1- 125. doi:10.1080/10408440802272158

16. KatzKD. Organophosphate Toxicity. Availableat: http:// emedicine. medscape.com/article/167726-overview. Accessed on 14th March2014.

17. SoferS, Tal T, Shahak E. Carbamate and organophosphate poisoning in early childhood. PediatrEmerg Care 1989, 5:222-225.doi: 10.1097/00006565-198912000-00005

18. Mortensen MD. Management of acute childhood poisoning caused by selected insectisides and herbicides. PediatrClin North Am1986. $33: 421-445$

19. NandN, Aggarwal HK, Bharti K, Chakrabarti D. Organophosphate induced delayed neuropathy. J Assoc Physicians India. 2007; 55:72-73.

20. Meena SS, Kumar TVR. OrganophosphorousPoisoningand Neonatal cholinergic syndrome. Indian Journal of Applied Research 2014; 4(2): 36-37.

21. DuToitPW, Muller FO, Van Tonder WM, UngererMJ. Experience with the intensive care management of organophosphate insecticide poisoning.SAfrMed J. 1981; 60:227-229.

22. Merrill DG, MihmFG. Prolonged toxicity of organophosphate poisoning.Crit Care Med. 1982;10(8): 550-551.doi: 10.1097/00003246- 198208000-00015.

23. Bardin PG, Van Eeden SF. Organophosphate poisoning: grading the severity and comparing treatment between atropine and glycopyrrolate.Crit Care Med. 1990;18(9) :956-60.

24. Balali-Mood M, Mohammed-Hossain A, Hasan A. Effect ofhigh doses of sodium bicarbonate in acute Organophosphoruspesticide poisoning. Paper presented in SACTRC workshop,August 2004, Colombo, SriLanka.

25. Pajoumand A, Shadnia S, Rezaie A, Abdi M, Abdollahi M. Benefits of magnesium sulphate in the management of acutehuman poisoning by organophosphorus insecticides. HumExpToxicol 2004; 23:565-69. 
Bangladesh Crit Care J September 2020; 8 (2): 120-123

26. GuvenM, Sungur M, EserB, SartI, FezviA. The effects ofFresh Frozen Plasma on Cholinesterase Levels and Outcomesin Patients with Organophosphate Poisoning. J ToxicolClinToxicol2004; 42; $617-23$.

27. RanjbarA,Solhi H, MashayekhiFJ, SusanabdiA,RezaieA,Abdollahi M. Oxidative stress in acute human poisoning withorganophosphorusinsectides: a case control study. EnvironToxicolPharmacol2005; 20:88-91.

28. SogorbMA, VilanovaE, Carrera V. Future applications of phosphotriesterases in the prophylaxis and treatment of organophosphorusinsectide and nerve agent poisoning. ToxicolLett $2004 ; 151: 219-33$
29. LallementG, Baubicohn D, ClarenconD, et al. Review of the value of galyclidine (GK-11) as adjuvant medication toconventional treatments of organophosphate poisoning:primate experiments mimicking various scenarios of militaryor terrorist attack by soman. Neurotoxicology 1999; 20:675-84 